\title{
Quenching oscillations in combustion instabilities using model-based closed-loop multiplicative control
}

\author{
F. Bouziani, I.D. Landau, R.R. Bitmead \\ Laboratoire d'Automatique de Grenoble, ENSIEG BP 46, 38402 Saint-Martin d'Hères, France \\ $\dagger$ Department of Mechanical \& Aerospace Engineering, University of California, San Diego, La Jolla CA 92093-0411, USA
}

\begin{abstract}
This papers deals with active control of combustion instabilities through measurement and feedback of pressure oscillations. The measurement is used to construct a multiplicative feedback control. The aim of such a control is to quench the oscillations associated with the instability associated with lean pre-mixed combustion. This quenching is analyzed using the Krylov-Bogoliubov approach applied to a tractable gray-box model of the underlying process and fitted using experimental data. A linear and a nonlinear feedback law are considered and conditions for quenching the oscillations established. Both give successful results verified by the simulation tests.
\end{abstract}

Index Terms - active control, combustion instability, nonlinear oscillating systems, Krylov-Bogoliubov method.

\section{INTRODUCTION}

Thermo-acoustic combustion instabilities are dynamic phenomena that manifest themselves through strong selfsustained oscillations. They cause serious degradation of, particularly, pollution performance in combustion turbomachinery systems such as gas turbine powerplants and jet engines. These instabilities occur in lean premixed combustion and effectively dispel the low-pollution properties of such operating regimes. Physically the instabilities are highly nonlinear phenomena, that can be explained by a positive feedback coupling between the thermal heat-release process and the acoustics of the combustion chamber. The positive feedback coupling leads the creation of nonlinear limit cycles, which can be sometimes characterized by coexistence of oscillations at several distinct non-harmonic frequencies.

From a practical perspective, the modulation of a fuel flow fraction into the combustor is possible as a control input. This modulation has been widely tested in experiments as a candidate for active control for suppressing the combustion instability [1], [2]. In the literature, several active control method have been proposed; an excellent overview of existing methods is given in [3]. Systematic design and implementation of active control requires a realistic low order model which exhibits the dominant dynamical effects. The parsimonious modelling of such nonlinear systems is an extremely difficult task, given that high-order, physics-based computational fluid dynamical codes can be unreliable in demonstrating the phenomenon well. One particular feature which the model should capture is the simultaneous coexistence of two non-harmonic oscillating modes, [4], [5].

In the literature, three approaches to combustion instability modeling are considered. The first is white-box modelling, which derives from physical theory directly and relies on partial differential equations describing the reacting-flow fluid dynamics with boundary conditions given by the combustor geometry. The complexity of this task is evident in the difficulties evident in computational verification of the observed phenoena. To develop a low-order model of use for control, Galerkin projection, averaging and truncation are used in [6], yielding a low order model with two oscillating modes. This model was used in [7] for controller design of a simple case study (The frequencies of both modes are 1 and 2.). The second approach, black-box modelling uses data directly with system identification methods [8], [9] often with model structures which have no physical interpretation. The third approach, grey-box modelling uses physical insight to determine the model structure and the input/output data to fit the parametrized model. This approach is more attractive inasmuch as it represents the middle ground between white-box and black-box approaches. In [10], Peracchio and Proscia proposed a low order model resulting from several physical investigations. The model was characterized by a linear resonator in feedback with a delay and a nonlinear static function. By using the greybox approach and in order to explain the coexistence of two modes in the data, Dunstan and Bitmead [11] have extended the model through the inclusion of and additional third-harmonic resonance. In [12], the relationship between the occurrence of oscillations in combustion instabilities and the van der Pol equation has been discussed, and used to derive a model. In order to make modelling more realistic in [13], the model was generalized by incorporating delay and filtering. The model was successfully analyzed using the Krylov-Bogoliubov (K-B) method (detailed in [14], [15], [16], [17], [18]). This analysis showed that the model has the ability to capture a number of important distinct phenomena observed in practice, including the coexistence of two modes oscillating called the simultaneous self-sustained oscillations phenomenon, which can occur with a frequency ratios different from those demonstrated in models earlier; 1,3 and $\frac{1}{3}$.

The best known control method in active control of periodic oscillations is phase-shift control. This method feeds back the fundamental frequency component of the pressure measurement with an appropriate phase shift in order to reduce or to quench the oscillations. In the design of this controller, generally the combustion instability is considered as a single resonator. The controller is implemented for the 
resonant frequency with a bandpass filter which eliminates all other (and therefore regarded as uncontrolled) frequencies. The control method gives successful results in many applications [2]. However, at some operating conditions this controller can induce excitations at other non-controlled frequencies. Their are still many issues to be resolved with such a control design.

The present work is a continuation of [13]. The possibility of quenching oscillations by multiplicative feedback feedback control will be investigated. The multiple-resonator model model presented in [13] will be considered as the base for the study of oscillation quenching in combustion instabilities. However in order to illustrate the methodology a single resonator model will be considered first. The previous analysis [12], [13] indicates clearly that the K-B method is a powerful analysis method for the combustion instabilities. For this reason, the method will be considered in this paper as the main tool for the description of the system and its behavior.

\section{FiRST K-B APPROXIMATION FOR AUTONOMOUS MULTI-RESONATOR SYSTEMS}

Consider a system with $n$ resonators described by differential equations of the form,

$$
\frac{d^{2} x_{j}}{d t^{2}}+\omega_{j}^{2} x_{j}=\varepsilon f_{j}\left(x, \frac{d x}{d t}\right), \quad(j=1,2, \ldots, n),
$$

where $x=\left\{x_{1}, \ldots, x_{n}\right\}, \frac{d x}{d t}=\left\{\frac{d x_{1}}{d t}, \ldots, \frac{d x_{n}}{d t}\right\}$ and $\varepsilon$ is a small parameter. For the $j$ th resonator, the first K-B approximation (for more details see Chapter 2 of [17]) proposes the solution

$$
x_{j}=a_{j} \cos \left(\psi_{j}\right),
$$

where $\psi_{j}=\omega_{j} t+\theta_{j}, a_{j}$ and $\theta_{j}$ are slowly time-varying functions obeying the equations

$$
\left\{\begin{array}{l}
\frac{d a_{j}}{d t}=-\frac{\varepsilon}{2 \omega_{j}} H_{j j}\left(a_{1}, \ldots, a_{n}, \theta_{1}, \ldots, \theta_{n}\right), \\
\frac{d \theta_{j}}{d t}=-\frac{\varepsilon}{2 \omega_{j} a_{j}} G_{j j}\left(a_{1}, \ldots, a_{n}, \theta_{1}, \ldots, \theta_{n}\right),
\end{array}\right.
$$

with $H_{j j}$ and $G_{j j}$ obtained from the function $f_{j}\left(x, \frac{d x}{d t}\right)$ by substituting

$$
\left\{\begin{array}{l}
x_{k}=a_{k} \cos \left(\omega_{k} t+\theta_{k}\right), \\
\frac{d x_{k}}{d t}=-a_{k} \omega_{k} \sin \left(\omega_{k} t+\theta_{k}\right),
\end{array} \quad(k=1,2, \ldots, n)\right.
$$

and by setting it in the form

$$
\begin{aligned}
& f_{j}\left(a_{1} \cos \left(\omega_{1} t+\theta_{1}\right), \ldots, a_{n} \cos \left(\omega_{n} t+\theta_{n}\right),\right. \\
& \left.\quad-a_{1} \omega_{1} \sin \left(\omega_{1} t+\theta_{1}\right), \ldots,-a_{n} \omega_{n} \sin \left(\omega_{n} t+\theta_{n}\right)\right) \\
& =H_{j j} \sin \left(\omega_{j} t+\theta_{j}\right)+G_{j j} \cos \left(\omega_{j} t+\theta_{j}\right) \\
& \quad+\sum_{\omega_{j} \nsucc \omega_{\ell}}^{r}\left(H_{\ell j} \sin \left(\omega_{\ell} t+\theta_{\ell}\right)+G_{\ell j} \cos \left(\omega_{\ell} t+\theta_{\ell}\right)\right),
\end{aligned}
$$

where $\omega_{\ell}$ and $\theta_{\ell}$ are integer linear combinations of $\omega_{1}, \ldots, \omega_{n}$ and $\theta_{1}, \ldots, \theta_{n}$, respectively, and $r$ is the number of possible integer linear combinations of $\omega_{1}, \ldots, \omega_{n}$ different from $\omega_{j}$. For $x_{j}$ the coefficients of the fundamental term in (5) are used and the all other terms are eliminated.

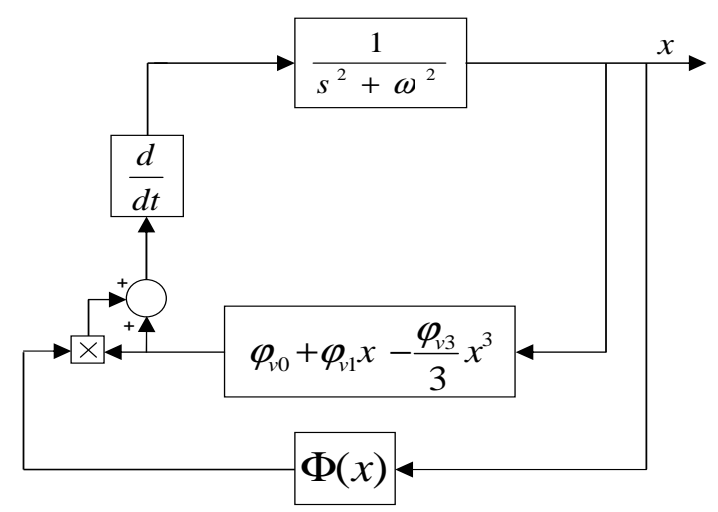

Fig. 1. Closed-loop control for model based on a generalized Van der Pol equation

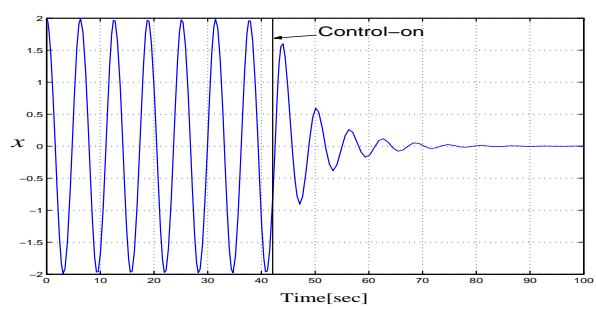

Fig. 2. Simulation test of quenching oscillations on a model described by a generalized van der Pol equation, where $K=1, \varphi_{v 0}=0.45, \varphi_{v 1}=\varphi_{v 1}=0.1$ and $\omega=1$.

\section{Simple CASE STUdY: MODEL BASED ON A GENERALIZED VAN DER POL EQUATION}

In this section a reduced-order model with one single resonator and without time delay (corresponding to a generalized van der Pol equation) will be considered to illustrate the potential effectiveness of the closed-loop multiplicative control. This output-feedback control is introduced into the model by multiplying a function of the output $x$ to capture the effect of fuel flow modulation on the heat release rate. The control strategy is presented in the block diagram shown in Figure 1 and it is characterized by the following differential equation.

$$
\ddot{x}+\omega^{2} x=\frac{d}{d t}\left\{(1+\Phi(x))\left(\varphi_{v 0}+\varphi_{v 1} x-\frac{\varphi_{v 3}}{3} x^{3}\right)\right\},
$$

where; $\omega$ is the natural frequency, $\varphi_{v 1}$ and $\varphi_{v 3}$ are arbitrary positive constants, $\varphi_{v 0}$ is an arbitrary constant different from zero and the feedback law $\Phi(x)$ is a polynomial function of $x$.

It is well known that for the uncontrolled generalized van der Pol equation [14], [15], the solution $x$ is a selfsustained oscillation with steady frequency close to $\omega$ and with steady amplitude close to $2 \sqrt{\frac{\varphi_{v 1}}{\varphi_{v 3}}}$. In order to quench this oscillation, the control law must force the system (6) to be asymptotically stable at the origin. Hence, one considers the following lemma.

Lemma 1: For the following control low

$$
\Phi(x)=-K x-\frac{1}{\varphi_{v 0}}\left(\varphi_{v 1} x-\frac{\varphi_{v 3}}{3} x^{3}\right),
$$


where $K$ is a constant of the same sign of $\varphi_{v 0}$, the system (6) is locally asymptotically stable at the origin.

Proof: Introducing the expression (7) into the differential equation (6), one gets

$$
\begin{aligned}
\ddot{x}+\omega^{2} x= & -K \varphi_{v 0} \dot{x}-2 \varphi_{v 1}\left(K+\frac{\varphi_{v 1}}{\varphi_{v 0}}\right) x \dot{x} \\
& +\frac{4 \varphi_{v 3}}{3}\left(K+\frac{2 \varphi_{v 1}}{\varphi_{v 0}}\right) x^{3} \dot{x}-2 \frac{\varphi_{v 3}^{2}}{3 \varphi_{v 0}} x^{5} \dot{x}
\end{aligned}
$$

Expressing this equation in state equation form with $z_{1}=x$ and $z_{2}=\dot{x}$, one obtains

$$
\left\{\begin{array}{rl}
\dot{z}_{1}= & z_{2} \\
\dot{z}_{2}= & -\omega^{2} z_{1}-K \varphi_{v 0} z_{2}-2 \varphi_{v 1}\left(K+\frac{\varphi_{v 1}}{\varphi_{v 0}}\right) z_{1} z_{2} \\
& \quad+\frac{4 \varphi_{v 3}}{3}\left(K+\frac{2 \varphi_{v 1}}{\varphi_{v 0}}\right) z_{1}^{3} z_{2}-2 \frac{\varphi_{v 3}^{2}}{3 \varphi_{v 0}} z_{1}^{5} z_{2}
\end{array} .\right.
$$

Computation of the linearized system matrix around the origin gives

$$
A_{z}=\left[\begin{array}{lr}
0 & 1 \\
-\omega^{2} & -K \varphi_{v 0}
\end{array}\right],
$$

since by assumption $K$ and $\varphi_{v 0}$ have the same sign, the eigenvalues of matrix $A_{z}$ will have negative real part. By using Lyapunov's indirect method, one can deduce that the system is locally asymptotically stable at the origin.

The local asymptotical stability at the origin implies that quenching of the oscillation is possible and can occur in a local domain around the origin which can be estimated [19]. This quenching is illustrated by the simulation test presented in Figure 2.

\section{COMBUSTION INSTABILITY MODEL}

The model from [13] is chosen as a basis for the development of effective control methods for quenching both oscillation modes present in combustion instabilities. However, the multiplicative effect of the control action must be included in the representation to capture the modulation of a fraction of the fuel flow $u$ into the combustion chamber and its consequent effect on the heat release rate [11], [5]. This leads to the following differential equations.

$$
\left\{\begin{array}{l}
\ddot{x}_{1}+\omega_{1}^{2} x_{1}=\frac{d}{d t} L P F\left\{(1+u)\left(\varphi_{v 0}+\varphi_{v 1} \dot{p}_{\tau}-\frac{\varphi_{v 3}}{3} \dot{p}_{\tau}^{3}\right)\right\}, \\
\ddot{x}_{2}+\omega_{2}^{2} x_{2}=\frac{d}{d t} L P F\left\{(1+u)\left(\varphi_{v 0}+\varphi_{v 1} \dot{p}_{\tau}-\frac{\varphi_{v 3}}{3} \dot{p}_{\tau}^{3}\right)\right\} .
\end{array}\right.
$$

where; $\varphi_{v 0}$ is an arbitrary constant, $\varphi_{v 1}$ and $\varphi_{v 3}$ are arbitrary negative constants, $\tau$ is a transport time delay from nozzle to flame surface, $L P F$ is the transfer function operator of a low pass filter, $p=x_{1}+x_{2}$ is the downstream pressure perturbation at the burning plane, $\dot{p}_{\tau}$ is the output of the delay-plus-differentiator block, and $q=\varphi_{v 0}+\varphi_{v 1} \dot{p}_{\tau}-\frac{\varphi_{v 3}}{3} \dot{p}_{\tau}^{3}$ is the flame heat release rate. Figure 3 shows a simulation test with an appropriate choice of parameter values for coexistence of two non-harmonic oscillations. For the following simulations, these parameter values will be used.

From an analytical point of view, the presence of the delay $\tau$ and the filter $L F P$ leads to some difficulties, which will be the subject of two assumptions proposed (and discussed more fully) in [13].
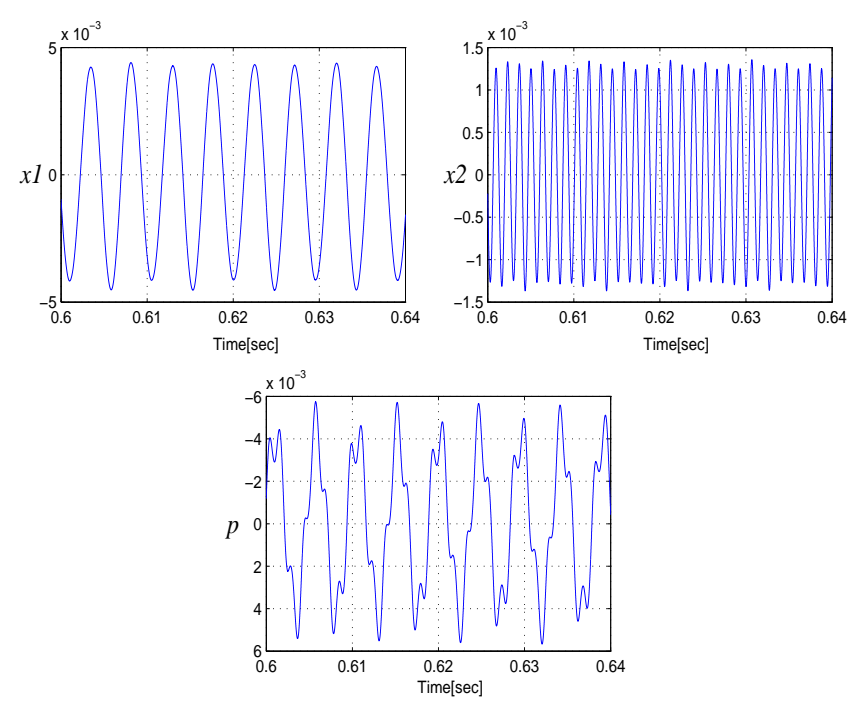

Fig. 3. Coexistence of two modes in the combustion instability model of [13], with $\omega_{1}=2 \pi \times 210, \omega_{2}=2 \pi \times 740, \varphi_{v 0}=0.45, \varphi_{v 1}=-0.135$, $\varphi_{v 3}=-5.4 \times 10^{-3}, L P F=\frac{2 \pi \times 500}{s+2 \pi \times 500}$ and $\tau=4.8 \times 10^{-3}$

Assumption 1: For small values of the time delay $\tau$, the oscillatory components of the output of delay block can approximated by

$$
\begin{aligned}
& \dot{x}_{1}(t-\tau) \approx \omega_{1} a_{1} \cos \left(\omega_{1} t+\theta_{1}+\frac{\pi}{2}-\omega_{1} \tau\right) \\
& \dot{x}_{2}(t-\tau) \approx \omega_{2} a_{2} \cos \left(\omega_{2} t+\theta_{2}+\frac{\pi}{2}-\omega_{2} \tau\right) .
\end{aligned}
$$

Assumption 2: The output of the linear low pass filter for sinusoidal inputs with slowly time-varying amplitudes and phases can be approximated by

$$
\operatorname{LPF}(a \cos (\omega t+\theta)) \approx G(\omega) a \cos (\omega t+\theta-\phi(\omega))
$$

where $a, \omega$ and $\theta$ are the amplitude, the frequency and the phase of input, respectively, $G(\omega)$ and $\phi(\omega)$ are the gain and the phase at frequency $\omega$ introduced by the filter, respectively.

\section{Closed-LOOP CONTROL OF COMBUStion INSTABILITIES}

In this section, the possibility of dealing with the instabilities by linear and nonlinear feedback law is analyzed. The basic idea is to use the pressure measurement $p$ in feedback for quenching both oscillations simultaneously. Two types of control law are considered, one linear the other nonlinear, for the calculation of the input to the multiplicative control actuator.

To study the effects of each control we use the K-B method. The following assumption is proposed concerning the validity of the K-B approximation.

Assumption 3: Let $a_{1}$ and $a_{2}$ be the amplitudes of the oscillations $x_{1}$ and $x_{2}$ obtained from K-B approximation. If $a_{1}$ and $a_{2}$ are asymptotically locally (globally) stable at the origin, then the original system is asymptotically locally stable at the origin. 


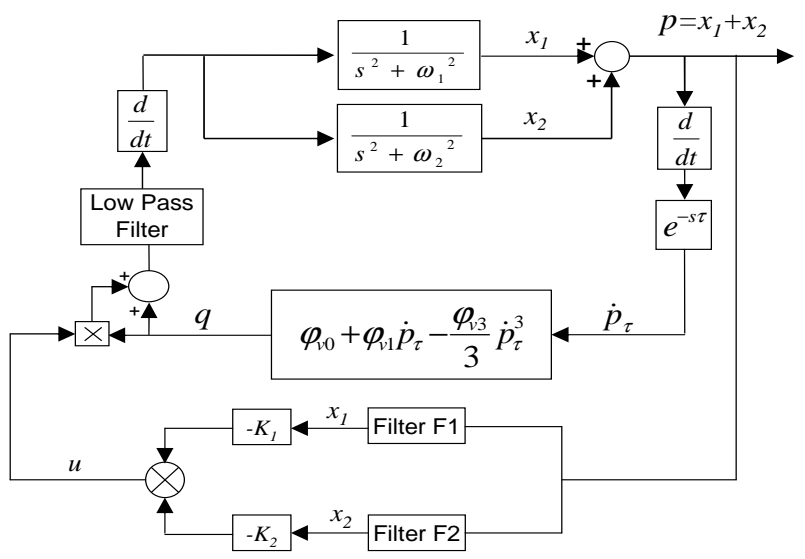

Fig. 4. Block diagram of the linear feedback controller

\section{A. Linear feedback: Compensation of negative damping}

For a linear feedback the control law will be of the form $u=-K_{1} x_{1}-K_{2} x_{2}$, where $K_{1}$ and $K_{2}$ are gains. Unfortunately, the pressure measurement $p=x_{1}+x_{2}$ and to obtain $x_{1}$ and $x_{2}$, band pass filters should be considered. In what follows we will assume that $x_{1}$ and $x_{2}$ are available in order to explore the possibility of stabilizing the system with a linear feedback. This control strategy corresponds to the block diagram shown in Figure 4. The aim of such a control is to compensate for the negative damping introduced by the physical loop and to introduce another positive damping, which causes the system (9) to become asymptotically locally stable at the origin. The gains $K_{1}$ and $K_{2}$ must be tuned to accommodate the phase delay and to ensure closed-loop stability.

Lemma 2: Consider the system (9) and linear control law above subject to the previous assumptions. Then provided

$$
\left\{\begin{array}{l}
K_{1} \varphi_{v 0} \cos \left(\phi\left(\omega_{1}\right)\right)>\omega_{1} \varphi_{v 1} \sin \left(\phi\left(\omega_{1}\right)+\omega_{1} \tau\right), \\
K_{2} \varphi_{v 0} \cos \left(\phi\left(\omega_{2}\right)\right)>\omega_{2} \varphi_{v 1} \sin \left(\phi\left(\omega_{2}\right)+\omega_{2} \tau\right),
\end{array}\right.
$$

the system is locally asymptotically stable at the origin.

Proof: We make the following identifications in the K-B approximation representation (1) with $\varepsilon=1$.

$$
f_{1}=f_{2}=f=\frac{d}{d t} L P F\left\{(1+u)\left(\varphi_{\nu 0}+\varphi_{v 1} \dot{p}_{\tau}-\frac{\varphi_{\nu 3}}{3} \dot{p}_{\tau}^{3}\right)\right\} .
$$

As in [13], we introduce notations $x_{i}=a_{i} \cos \left(\omega_{i} t+\right.$ thet $\left._{i}\right)$ and $\dot{x}_{i}=-a_{i} \omega_{i} \sin \left(\omega_{i} t+\theta_{i}\right), i=1,2$ in (12). After trigonometric simplifications and using Assumptions 1 and 2, one obtains

$$
\begin{aligned}
& f \approx a_{1} G\left(\omega_{1}\right) \sin \left(\omega_{1} t+\theta_{1}\right)\left\{K_{1} \varphi_{v 0} \omega_{1} \cos \left(\phi\left(\omega_{1}\right)\right)-\varphi_{v 1} \omega_{1}^{2}\left[1-\frac{\varphi_{v 3}}{\varphi_{v 1}}\left(\frac{\left(\omega_{1} a_{1}\right)^{2}}{4}\right.\right.\right. \\
& \left.\left.\left.+\frac{\left(\omega_{2} a_{2}\right)^{2}}{2}\right)\right] \sin \left(\omega_{1} \tau+\phi\left(\omega_{1}\right)\right)\right\}-a_{1} G\left(\omega_{1}\right) \cos \left(\omega_{1} t+\theta_{1}\right)\left\{K_{1} \varphi_{v 0} \omega_{1}\right. \\
& \left.\times \sin \left(\phi\left(\omega_{1}\right)\right)+\varphi_{v 1} \omega_{1}^{2}\left[1-\frac{\varphi_{v 3}}{\varphi_{v 1}}\left(\frac{\left(\omega_{1} a_{1}\right)^{2}}{4}+\frac{\left(\omega_{2} a_{2}\right)^{2}}{2}\right)\right] \cos \left(\omega_{1} \tau+\phi\left(\omega_{1}\right)\right)\right\} \\
& +a_{2} G\left(\omega_{2}\right) \sin \left(\omega_{2} t+\theta_{2}\right)\left\{K_{2} \varphi_{v 0} \omega_{2} \cos \left(\phi\left(\omega_{2}\right)\right)-\varphi_{v 1} \omega_{2}^{2}\left[1-\frac{\varphi_{v 3}}{\varphi_{v 1}}\left(\frac{\left(\omega_{2} a_{2}\right)^{2}}{4}\right.\right.\right. \\
& \left.\left.\left.+\frac{\left(\omega_{1} a_{1}\right)^{2}}{2}\right)\right] \times \sin \left(\omega_{2} \tau+\phi\left(\omega_{2}\right)\right)\right\}-a_{2} G\left(\omega_{2}\right) \cos \left(\omega_{2} t+\theta_{2}\right)\left\{K_{2} \varphi_{v 0} \omega_{2}\right. \\
& \left.\sin \left(\phi\left(\omega_{2}\right)\right)-\varphi_{v 1} \omega_{2}^{2}\left[1-\frac{\varphi_{v 3}}{\varphi_{v 1}}\left(\frac{\left(\omega_{2} a_{2}\right)^{2}}{4}+\frac{\left(\omega_{1} a_{1}\right)^{2}}{2}\right)\right] \cos \left(\omega_{2} \tau+\phi\left(\omega_{2}\right)\right)\right\} \\
& +\sum_{\omega_{\ell} \approx \omega_{1} \wedge \omega_{2}}^{r}\left(H_{\ell}\left(a_{1}, a_{2}, \theta_{1}, \theta_{2}\right) \sin \left(\omega_{\ell} t+\theta_{\ell}\right)+G_{\ell}\left(a_{1}, a_{2}, \theta_{1}, \theta_{2}\right) \cos \left(\omega_{\ell} t+\theta_{\ell}\right)\right),
\end{aligned}
$$

where; $\omega_{\ell}$ is a linear combination of $\omega_{1}$ and $\omega_{2}, \theta_{\ell}$ is a linear combination of $\theta_{1}$ and $\theta_{2}, r$ is the number of possible linear combinations of $\omega_{1}$ and $\omega_{2}$ different from $\omega_{1}$ and $\omega_{2}$. After applying the rule (3) for amplitudes, this leads to the following approximations.

$$
\left\{\begin{array}{c}
\frac{d a_{1}}{d t}=\frac{G\left(\omega_{1}\right) \varphi_{v 1} \omega_{1} \sin \left(\omega_{1} \tau+\phi\left(\omega_{1}\right)\right)}{2} a_{1}\left[1-\frac{\varphi_{v 3}}{\varphi_{v 1}}\left(\frac{\left(\omega_{1} a_{1}\right)^{2}}{4}+\frac{\left(\omega_{2} a_{2}\right)^{2}}{2}\right)\right. \\
]-\frac{G\left(\omega_{1}\right) K_{1} \varphi_{v 0} \cos \left(\phi\left(\omega_{1}\right)\right)}{2} a_{1}, \\
\frac{d a_{2}}{d t}=\frac{G\left(\omega_{2}\right) \varphi_{v 1} \omega_{2} \sin \left(\omega_{2} \tau+\phi\left(\omega_{2}\right)\right)}{2} a_{2}\left[1-\frac{\varphi_{v 3}}{\varphi_{v 1}}\left(\frac{\left(\omega_{2} a_{2}\right)^{2}}{4}+\frac{\left(\omega_{1} a_{1}\right)^{2}}{2}\right)\right. \\
]-\frac{G\left(\omega_{2}\right) K_{2} \varphi_{v 0} \cos \left(\phi\left(\omega_{2}\right)\right)}{2} a_{2} .
\end{array}\right.
$$

The linearized system matrix around the origin becomes,

$$
\left[\begin{array}{ll}
\frac{G\left(\omega_{1}\right)}{2}\left(\varphi_{\nu 1} \omega_{1} \sin \left(\omega_{1} \tau+\phi\left(\omega_{1}\right)\right)-K_{1} \varphi_{\nu 0} \cos \left(\phi\left(\omega_{1}\right)\right)\right) & 0 \\
0 & \frac{G\left(\omega_{2}\right)}{2}\left(\varphi_{v 1} \omega_{2} \sin \left(\omega_{2} \tau+\phi\left(\omega_{2}\right)\right)-K_{2} \varphi_{v 0} \cos \left(\phi\left(\omega_{2}\right)\right)\right)
\end{array}\right]
$$

This matrix has two negative eigenvalues if the conditions (11) are satisfied. In this case, the amplitudes are locally asymptotically stable at the origin. Appealing to assumption 3 , one can concludes the result.

Local asymptotic stability at the origin implies that quenching the oscillations is possible, and occurs in a local domain which depends on the gains $K_{1}$ and $K_{2}$. When the values $K_{1}$ and $K_{2}$ increase, the domain of asymptotic stability around origin should also increase. For this reason and by comparison to damped second-order linear systems, the following gains are chosen in the subsequent simulations of this controller.

$$
\left\{\begin{array}{l}
K_{1}=\max \left(\frac{2 \eta \omega_{1}+\varphi_{v 1} \omega_{1} G\left(\omega_{1}\right) \sin \left(\phi\left(\omega_{1}\right)+\omega_{1} \tau\right)}{\varphi_{10} G\left(\omega_{1}\right) \cos \left(\phi\left(\omega_{1}\right)\right)}, 0\right) \\
K_{2}=\max \left(\frac{2 \eta \omega_{2}+\varphi_{01} \omega_{2} G\left(\omega_{2}\right) \sin \left(\phi\left(\omega_{2}\right)+\omega_{2} \tau\right)}{\varphi_{v 0} G\left(\omega_{2}\right) \cos \left(\phi\left(\omega_{2}\right)\right)}, 0\right)
\end{array},\right.
$$

where $\eta$ is the desired damping factor.

We consider two scenarios for simulation testing of this controller. In the first, the control is applied at the appearance of oscillations in $x_{1}$ or $x_{2}$. This is illustrated by a simulation test executed with $\eta=0.05\left(K_{1}=0\right.$ and $\left.K_{2}=4051\right)$ and presented in Figure 5. One can observe from the Figure 5, that this control law requires a very weak control signal $u$, noting that this depends on the ratio $\frac{\varphi_{v 1}}{\varphi_{v 0}}, c . f$ (13). The second scenario is where the control is applied after that the oscillations in $x_{1}$ and $x_{2}$ have already reached steadystate operation. Compared with the previous scenario, this requires sufficient control action to effect damping but also a need not to violate the validity of the approximations including linearization. This is illustrated by a simulation test with $\eta=0.1\left(K_{1}=160\right.$ and $\left.K_{2}=5700\right)$ and is presented in Figure 6.

This linear strategy under the conditions (11) offers local asymptotic stability, which makes quenching possible, but without any guarantee in a larger domain. However to effectively implement such quenching, the problem of obtaining $x_{1}$ and $x_{2}$ ba appropriate filtering has to be solved (work in progress). It would be preferable if the amplitudes $a_{1}$ and $a_{2}$ were to be globally stable. For this a non-linear close loop feedback must be considered. 

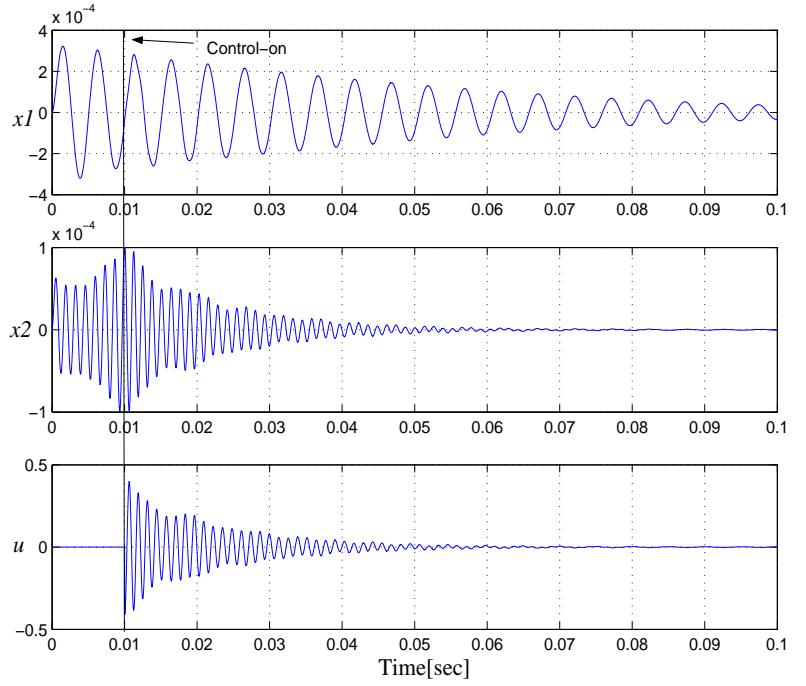

Fig. 5. Linear feedback control applied at the first appearance of the oscillations.
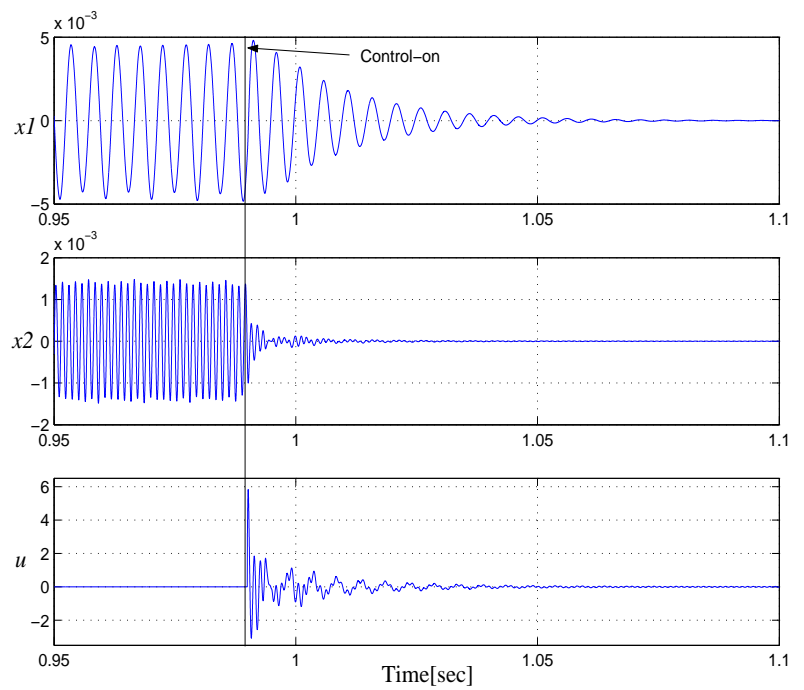

Fig. 6. Linear feedback control applied after the oscillations have reached steady state.

\section{B. Non-linear feedback: feedback linearization}

In this section, the pressure measurement $p$ is differentiated and delayed with a delay $\tau$ to obtain $\dot{p}_{\tau}$, which is introduced into a non-linear function $\Phi$, to obtain a control law $u=\Phi\left(p, \dot{p}_{\tau}\right)$. This control strategy is explained in the block diagram shown in Figure 7. This control law will be used to add damping and to compensate the physical feedback caused by the coupling between the thermal heat-release process and the acoustics of the combustion chamber. If such a control is designed, the system will be asymptotically stable at the origin and the quenching of oscillations will occur. This can be interpreted also as a feedback linearization which in addition stabilizes the system.For this reason, one considers the following lemma

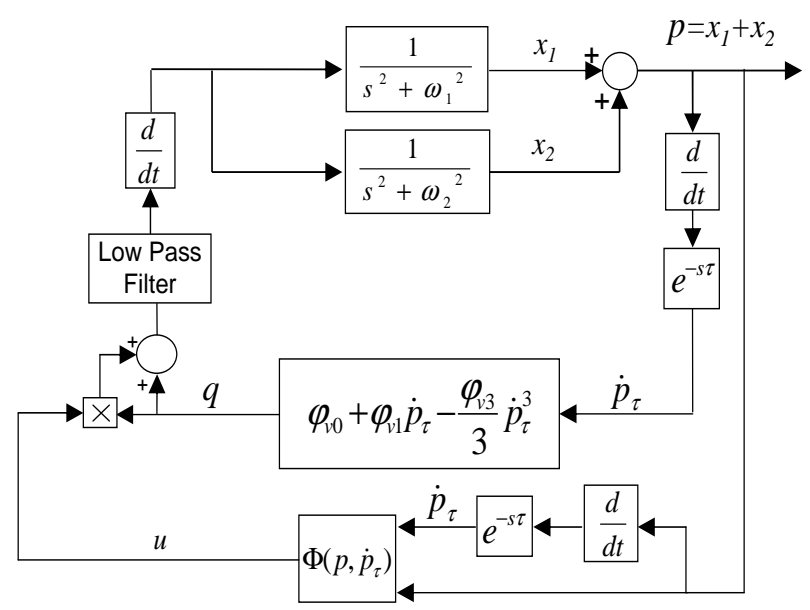

Fig. 7. Block diagram of non-linear feedback.

Lemma 3: For the control law

$$
\Phi\left(p, \dot{p}_{\tau}\right)=-K p-\frac{1}{\varphi_{v 0}}\left(\varphi_{v 1} \dot{p}_{\tau}-\frac{\varphi_{v 3}}{3} \dot{p}_{\tau}^{3}\right),
$$

where $K$ is a constant satisfying the conditions

$$
\left\{\begin{array}{l}
K \varphi_{v 0} \cos \left(\phi\left(\omega_{1}\right)\right)>0 \\
K \varphi_{v 0} \cos \left(\phi\left(\omega_{2}\right)\right)>0
\end{array}\right.
$$

the system is locally asymptotically stable at the origin.

Proof: Replacing the function (14) in (9) yields,

$$
\left\{\begin{aligned}
\ddot{x}_{1}+\omega_{1}^{2} x_{1}=\frac{d}{d t} L P F\{ & \varphi_{v 0}-K \varphi_{v 0} p-K \varphi_{v 1} p \dot{p}_{\tau}+K \frac{\varphi_{v 3}}{3} p \dot{p}_{\tau}^{3} \\
& \left.-\frac{\varphi_{v 1}^{2}}{\varphi_{v 0}} \dot{p}_{\tau}^{2}+\frac{2 \varphi_{v 1} \varphi_{v 3}}{3 \varphi_{v 0}} \dot{p}_{\tau}^{4}-\frac{\varphi_{v 3}^{2}}{9 \varphi_{v 0}} \dot{p}_{\tau}^{6}\right\}, \\
\ddot{x}_{2}+\omega_{2}^{2} x_{2}=\frac{d}{d t} L P F\{ & \varphi_{v 0}-K \varphi_{v 0} p-K \varphi_{v 1} p \dot{p}_{\tau}+K \frac{\varphi_{v 3}}{3} p \dot{p}_{\tau}^{3} \\
& \left.-\frac{\varphi_{v 1}^{2}}{\varphi_{v 0}} \dot{p}_{\tau}^{2}+\frac{2 \varphi_{v 1} \varphi_{v 3}}{3 \varphi_{v 0}} \dot{p}_{\tau}^{4}-\frac{\varphi_{v 3}^{2}}{9 \varphi_{v 0}} \dot{p}_{\tau}^{6}\right\} .
\end{aligned}\right.
$$

Therefore, for the K-B approximation (1) with $\varepsilon=1$ one may consider the following choice.

$$
\begin{aligned}
f_{1}=f_{2}=f=\frac{d}{d t} L P F & \left\{\varphi_{v 0}-K \varphi_{v 0} p-K \varphi_{v 1} p \dot{p}_{\tau}+K \frac{\varphi_{v 3}}{3} p \dot{p}_{\tau}^{3}\right. \\
& \left.-\frac{\varphi_{v 1}^{2}}{\varphi_{v 0}} \dot{p}_{\tau}^{2}+\frac{2 \varphi_{v 1} \varphi_{v 3}}{3 \varphi_{v 0}} \dot{p}_{\tau}^{4}-\frac{\varphi_{v 3}^{2}}{9 \varphi_{v 0}} \dot{p}_{\tau}^{6}\right\} .
\end{aligned}
$$

Introducing $x_{i}=a_{i} \cos \left(\omega_{i} t+\theta_{i}\right)$ and $\dot{x}_{i}=-a_{i} \omega_{i} \sin \left(\omega_{i} t+\theta_{i}\right)$, $i=1,2$, in (16) and after trigonometric simplifications and using assumption 2 , one obtains the expression

$f \approx K \varphi_{v 0} \omega_{1} G\left(\omega_{1}\right) a_{1}\left[\cos \left(\phi\left(\omega_{1}\right)\right) \sin \left(\omega_{1} t+\theta_{1}\right)-\sin \left(\phi\left(\omega_{1}\right)\right) \cos \left(\omega_{1} t+\theta_{1}\right)\right]$ $+K \varphi_{v 0} \omega_{2} G\left(\omega_{2}\right) a_{2}\left[\cos \left(\phi\left(\omega_{2}\right)\right) \sin \left(\omega_{2} t+\theta_{2}\right)-\sin \left(\phi\left(\omega_{2}\right)\right) \cos \left(\omega_{2} t+\theta_{2}\right)\right]$ $+\sum_{\omega_{\ell} \nLeftarrow \omega_{1} \wedge \omega_{2}}^{r}\left(H_{\ell}\left(a_{1}, a_{2}, \theta_{1}, \theta_{2}\right) \sin \left(\omega_{\ell} t+\theta_{\ell}\right)+G_{\ell}\left(a_{1}, a_{2}, \theta_{1}, \theta_{2}\right) \cos \left(\omega_{\ell} t+\theta_{\ell}\right)\right)$.

Applying the rule (3) for the amplitudes leads to the following approximations.

$$
\left\{\begin{aligned}
\frac{d a_{1}}{d t} & =-\frac{1}{2} G\left(\omega_{1}\right) K \varphi_{v 0} \cos \left(\phi\left(\omega_{1}\right)\right) a_{1}, \\
\frac{d a_{2}}{d t} & =-\frac{1}{2} G\left(\omega_{2}\right) K \varphi_{v 0} \cos \left(\phi\left(\omega_{2}\right)\right) a_{2} .
\end{aligned}\right.
$$

These are linear differential equations for the uncoupled amplitudes $a_{1}$ and $a_{2}$. The amplitudes are globally asymptotically stable at origin if the conditions (15) are satisfied. Appealing to the assumption 3, one deduces the result. 

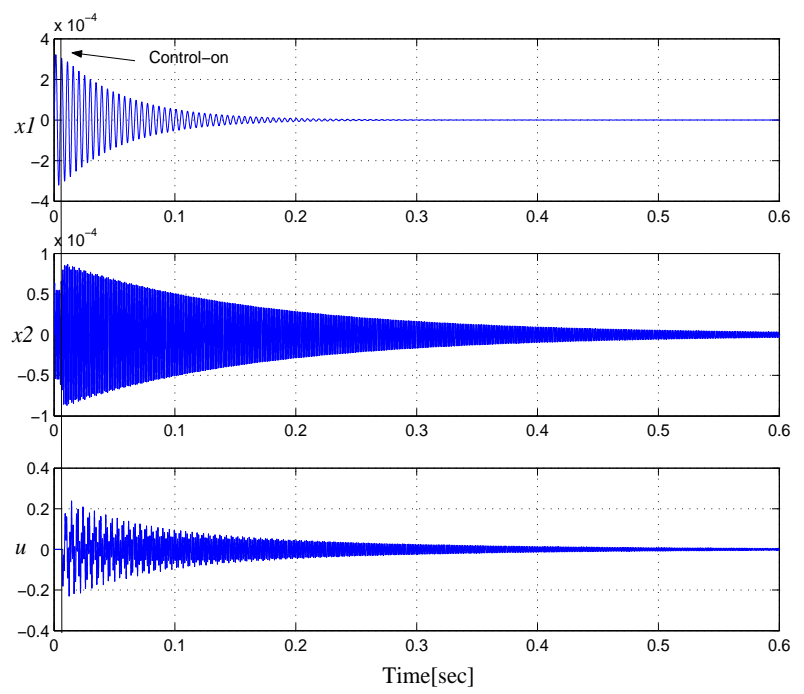

Fig. 8. Linear feedback control applied at the first appearance of oscillations.
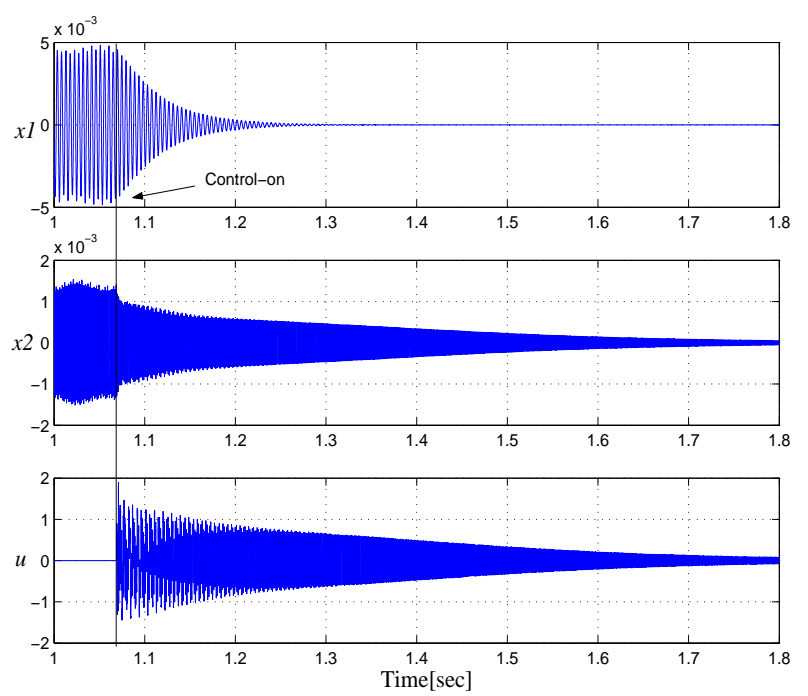

Fig. 9. Non-linear feedback control applied after the oscillation has reached steady-state.

This strategy has been tested in simulation, with gain $K=$ 100 for the two scenarios as before. First, when the control is applied at the appearance of oscillations in $x_{1}$ or $x_{2}$. This is presented in Figure 8. Then when the control is applied after that the oscillations of $x_{1}$ and $x_{2}$ have already reached steady-state operation. This is presented in Figure 9. One can note that compared to linear feedback, the quenching of oscillations is very slow. On the other hand, the control amplitude is smaller.

The satisfaction of conditions (15) on parameter $K$, is completely possible when the product $\cos \left(\phi\left(\omega_{1}\right)\right) \cos \left(\phi\left(\omega_{2}\right)\right)$ is strictly positive. However, when the product is negative, it is difficult and sometimes impossible to find a value $K$ satisfying (15). The problem can be avoided by using the Filters F1 and F2 (the same Filters used in the linear feedback) which allows the replacement of $K p$ by $K_{1} x_{1}+K_{2} x_{2}$ in the control

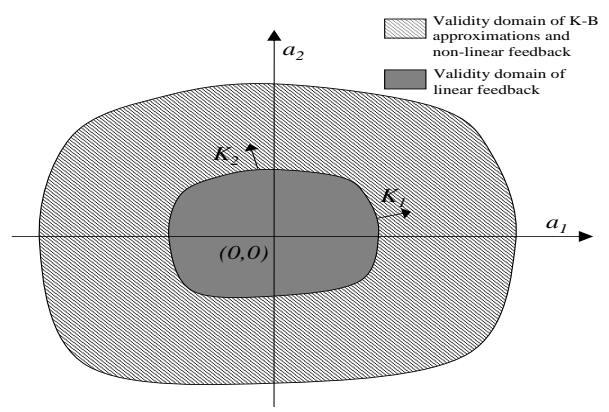

Fig. 10. Comparison between the computed quenching domains of both feedback controllers.

law to obtain the following conditions which are easier to satisfy.

$$
\left\{\begin{array}{l}
K_{1} \varphi_{v 0} \cos \left(\phi\left(\omega_{1}\right)\right)>0 \\
K_{2} \varphi_{v 0} \cos \left(\phi\left(\omega_{2}\right)\right)>0 .
\end{array}\right.
$$

\section{COMPARISON BETWEEN THE CONTROL STRATEGIES}

The nonlinear control law yields asymptotic local stability at the origin. The domain of amplitudes $a_{1}$ and $a_{2}$ where quenching oscillations is guaranteed, is delimited by the boundary of validity the K-B method applied to (9). For the linear feedback law, the quenching domain is limited not just by the validity of the K-B method, but also by the local limitations on the gain values $K_{1}$ and $K_{2}$ of feedback loop (Figure 10). Additionally, the nonlinear feedback requires less restriction on the delay value, since it does not use the Assumption 1. It is however important to note however, that both control strategies are predicated on having good knowledge of the parameters values in the combustion instability model, particularly of the value of the delay $\tau$.

\section{CONCLUSION}

This paper presents control methodologies for quenching oscillations in combustion instabilities based on a tractable model derived elsewhere, which exhibits the coexistence of two oscillating modes observed in combustion instabilities. The K-B method has been considered as a main tool for analysis and conditions for quenching have been established.

Control by linear feedback was firstly considered. The study showed that quenching oscillations is possible in a local stability domain, which depends on the feedback gains. The simulation tests show that quenching was successfully, requiring more control efforts in the case of oscillations which had reached steady state. To increase the local stability domain, the non-linear feedback was considered as an alternative solution. This feedback also quenches oscillations in a local stability domain, but one which depends solely on the domain of validity of K-B approximations. In other word, the feedback offers a stability domain larger than the domain obtained by linear feedback. Good knowledge of model parameters is necessary for quenching with either feedback approach.

Further work will focus on the robustness of these approaches with respect to model parameters uncertainties as well as a quantitative evaluation of the stability domain. 


\section{REFERENCES}

[1] G. Roy, Advances in Chemical Propulsion: Science to Technology. Boc Raton, Fl USA: CRC - Taylor \& Francis, 2001.

[2] A. Banaszuk, K. Ariyur, M. Krstic, and C. Jacobson, "An adaptive algorithm for control of combustion instability," Automatica, vol. 40, pp. 2155-2162, 2004.

[3] A. Annaswamy and A. Ghoniem, "Active control of combustion instability: Theory and practice," IEEE Control Systems Magazine, vol. 22, no. 6, pp. 37-54, Dec 2002.

[4] R. M. Murray, C. A. Jacobson, R. Casas, A. I. Khibnik, C. R. Johnson Jr, R. R. Bitmead, A. A. Peracchio, and W. M. Proscia, "System identification for limit cycling systems: a case study for combustion instabilities," American Control Conference, Philadelphia PA, pp. 2004-2008, 1998.

[5] W. J. Dunstan, R. R. Bitmead, and S. M. Savaresi, "Fitting nonlinear low-order models for combustion instability control," Control Engineering Practice, pp. 1301-1317, 2001.

[6] L. G. Paparizos and F. E. C. Culick, "The two-mode approximation to nonlinear acoustics in combustion chambers," Combustion Science and Technology, vol. 65, pp. 39-65, 1989.

[7] N. Al-Masoud and T. Singh, "Closed-loop amplitude modulation control of reacting premixed turbulent jet," IEEE Transactions on Control Systems Technology, vol. 13, pp. 1076-1082, 2005.

[8] T. Yi, M. Cornwell, and E. J. Gutmark, "Mean flow regulation of a high frequency combustion control valve based on pulse width modulation and system identification," American Control Conference, 2005.

[9] J. H. Uhm and S. Acharya, "Low-bandwith open-loop control of combustion instabity," Combustion and Flame, vol. 142, pp. 348-363, 2005.

[10] A. Peracchio and W. Proscia, "Nonlinear heat-release/acoustic model for thermoacoustic instability in lean premixed combustors," ASME J. Eng. Gas Turbines Power, vol. 121, no. 3, pp. 415-421, 1999.

[11] W. J. Dunstan, "System identification of nonlinear resonant systems," Ph.D. dissertation, University of California, San Diego, 2003.

[12] F. Bouziani, I. D. Landau, R. R. Bitmead, and A. Voda-Besançon, "An analytically tractable model for combustion instability," Conference on Decision and Control and European Control Conference, no. 44, 2005.

[13] _ "Analysis of a tractable model for combustion instability: the effect of delay and low pass filtering," Conference on Decision and Control-San Diego (accepted), no. 45, 2006.

[14] N. Bogoliubov and Y. Mitropolski, Asymptotic Methods in the Theory of Nonlinear Oscillations. New York: Hindustan Publishing Corp, Delhi, and Gordon and Breach, 1961.

[15] C. Hayashi, Nonlinear Oscillations in Physical Systems. New York: McGraw-Hill Book Co, 1964, (reprinted by Princeton University Press, 1985).

[16] P. S. Landa, Nonlinear Oscillations and Waves in Dynamical Systems. Kluwer, 1996.

[17] - Regular and Chaotic Oscillations, V. I. Babitsky and J. Wittenburg, Eds. New York: Springer, 2000

[18] I. D. Landau and R. R. Bitmead, "On the method of Krylov and Bogoliubov for the analysis of nonlinear oscillations," Mechanical and Aerospace Engineering Department, University of California, San Diego, 9500 Gilman Drive, La Jolla CA 92093-0411, USA, Tech. Rep., Jan 2004.

[19] H. K. khalil, Nonlinear Systems. New York: MacMillan, 1992. 